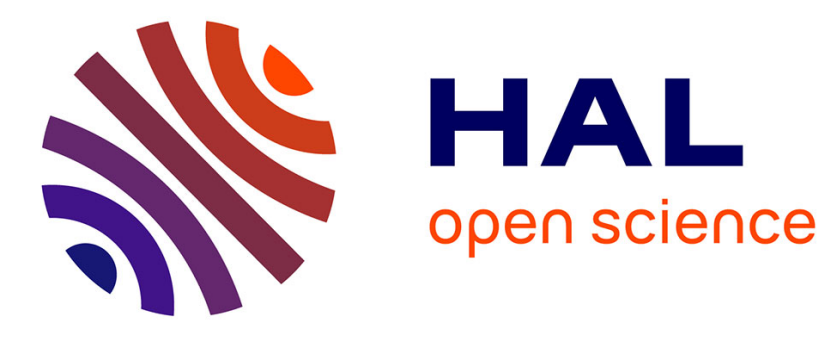

\title{
Control invariant partition for heterogeneous Multi-agent dynamical systems.
}

\author{
Minh Tri Nguyen, Cristina Stoica Maniu, Sorin Olaru
}

\section{To cite this version:}

Minh Tri Nguyen, Cristina Stoica Maniu, Sorin Olaru. Control invariant partition for heterogeneous Multi-agent dynamical systems.. 19th International Conference on Systems Theory, Control and Computing (ICSTCC), Oct 2015, CHEILE GRADISTEI, Romania. pp.354-359, 10.1109/ICSTCC.2015.7321319 . hal-01259477

\section{HAL Id: hal-01259477}

https://hal-centralesupelec.archives-ouvertes.fr/hal-01259477

Submitted on 16 Mar 2020

HAL is a multi-disciplinary open access archive for the deposit and dissemination of scientific research documents, whether they are published or not. The documents may come from teaching and research institutions in France or abroad, or from public or private research centers.
L'archive ouverte pluridisciplinaire HAL, est destinée au dépôt et à la diffusion de documents scientifiques de niveau recherche, publiés ou non, émanant des établissements d'enseignement et de recherche français ou étrangers, des laboratoires publics ou privés. 


\title{
Control invariant partition for heterogeneous Multi-Agent dynamical systems
}

\author{
M.T. Nguyen, C. Stoica Maniu, S. Olaru
}

\begin{abstract}
This paper aims to present a novel constructive framework for the functioning zone assignment of linear heterogeneous Multi-Agent dynamical systems, in order to improve the operation safety in a decentralized manner. The main contributions are related to partitioning the working space of this global system into multiple non-overlapping regions. The construction of the partition is mainly based on the desired position of each agent in a predefined formation and subject to constraints related to the local dynamics of the agents. Each region is assigned to one agent and subsequently guaranteed to be control invariant by applying an optimization-based decentralized control. A numerical example will be shown in order to prove the effectiveness of the proposed approaches.
\end{abstract}

Index Terms-Multi-agent dynamical systems, set-theoretic tools, decentralized control, collision avoidance constraints

\section{INTRODUCTION}

The attention for Multi-Agent dynamical Systems raised in the recent years due to their various applications, such as traffic control, robotics or spacecraft operations. For such applications, the collision avoidance is one of the most important features, being related to the mission safety of the whole Multi-Agent System (MAS). This problem becomes even more challenging when considering MAS of large dimensions, which are also affected by possible disturbances issued from the environment. In this context, centralized approaches are not appropriate to solve the collision avoidance problem due to the important computation and communication load. To overcome these drawbacks, decentralized approaches are mentioned (e.g. [1], [2], [3]) but without a complete stability proof in the presence of adversary constraints as the collision avoidance.

Recent results have been reported on the application of settheoretic and optimization tools to design the control with respect to a set of obstacle avoidance constraints [4] and further collision avoidance constraints for Multi-Agent dynamical systems [3]. The authors of [4] pointed out that regulating a system with respect to a set of obstacle avoidance constraints is equivalent to control the system around an equilibrium point which is on the boundary of its admissible domain, represented as a convex polyhedral region. In [3], the sets characterizing each agent are obtained and the collision avoidance constraints are guaranteed if these sets do not overlap. Furthermore, a feedback control with respect to the obstacle avoidance constraints for a single agent is proposed in [3].

In order to overcome the complexity of a centralized approach, a decentralized approach using the leader-follower

M.T. Nguyen, C. Stoica Maniu and S. Olaru are with Laboratoire des Signaux et Systèmes (L2S, UMR CNRS 8506), CentraleSupélec-CNRSUniversité Paris-Sud, Automatic Control Department, 3 rue Joliot Curie, F-91192, Gif-sur-Yvette cedex, France (e-mail: \{minhtri.nguyen; cristina.stoica; sorin.olaru\}esupelec.fr). structure is considered in the present study. In this approach, an agent can communicate only with its closest neighbors which allows to reduce the communication task. However, this requires that each agent is authorized to operate within a restricted zone, called functioning zone to ensure the collision avoidance. In addition, these zones do not intersect and their union defines a partition of the working space. Some notable recent results are reported on the application of Voronoi diagrams to partition the working space of MAS. In [5], the authors used the lifting map technique to construct a static Voronoi diagram for a set of points. This diagram is described in terms of solving an optimization problem. Another recent result in [6] employed an optimal control technique to obtain a Voronoi partition for a set of continuous-time dynamical agents and the dynamics of agents are employed in the Voronoi diagram construction.

In this context, the aim of the present paper is to propose a decentralized control strategy to deal with the collision avoidance constraints for a group composed of one leader agent and its followers. This strategy is based on the extension of controlled invariance results for linear time-invariant systems. The proposed approach is composed of two main steps. The first step concerns how to partition the working space into multiple non overlapping functioning zones. The second step is to design a control strategy to keep the agent operating in its functioning zone.

The remainder of the paper is organized as follows. The useful notations throughout this paper are briefly presented in Section II. Some important prerequisites and the problem formulation are presented in Section III. The control strategy to keep an agent operating within its functioning zone is proposed in Section IV, once a partition is determined. The partition construction subject to the dynamics of the agents is presented in Section V. Finally, numerical simulation results are illustrated in Section VI, followed by some concluding remarks and perspectives in Section VII.

\section{MAThematical nOtATions}

$0_{n, m}$ will represent a zero matrix in $\mathbb{R}^{n \times m}$. Given two matrices $P, Q \in \mathbb{R}^{n \times m}$, diag $\{P, Q\}$ and $\operatorname{col}\{P, Q\}$ denote respectively the block diagonal matrix and the block column matrix of $P$ and $Q . Q=Q^{\top} \succ 0$ implies that $Q$ is a symmetric strictly positive definite matrix.

$\mathcal{A} \oplus \mathcal{B}=\{a+b \mid a \in \mathcal{A}, b \in \mathcal{B}\}$ denotes the Minkowski sum of two sets $\mathcal{A}$ and $\mathcal{B}$. $\operatorname{Conv}\left\{v_{i}\right\}$ denotes the convex hull set of a given set of vertices $\mathcal{V}=\left\{v_{i} \mid v_{i} \in \mathbb{R}^{n}\right\}$. $\operatorname{int}(\mathcal{A})$ denotes the strict interior of the set $\mathcal{A}$. 
The set $\mathcal{C}(G)=\left\{x \in \mathbb{R}^{n} \mid G x \leq 0_{p, 1}, G \in \mathbb{R}^{p \times n}\right\}$ denotes the polyhedral proper cone.

The following definitions and results are recalled in order to facilitate the comprehension of this paper.

Definition 1. [7] Consider an autonomous linear system $x^{+}=$ $A x+w . A$ set $\mathcal{S}$ is called robustly positive invariant (RPI) for this system, if $x^{+} \in \mathcal{S}$ for all $x \in \mathcal{S}, w \in \mathcal{W}$, which is equivalent to $A \mathcal{S} \oplus \mathcal{W} \subseteq \mathcal{S}$.

Definition 2. [4] Given the system dynamics $x^{+}=A x+B u$, a set $\mathcal{S} \in \mathbb{R}^{n}$ is control invariant, if for any $x \in \mathcal{S}$ there exists a control action $u$ such that $A x+B u \in \mathcal{S}$. If $u=K x$ then $\mathcal{S}$ is linearly control invariant.

Definition 3. [8] A set $\mathcal{S}=\left\{x \in \mathbb{R}^{n} \mid G x \leq \theta\right\}$ is positively invariant with respect to the system dynamics $x^{+}=A x$ if and only if there exists a matrix $H \in \mathbb{R}^{n \times n}$ with non-negative elements, such that $G A=H G$ and $H \theta \leq \theta$.

Definition 4. A polyhedron $\mathcal{P}$ can be represented as a linear combination of its vertices $v_{i}$ and its rays $r_{i}$ i.e. $\mathcal{P}=\{x \in$ $\left.\mathbb{R}^{n} \mid x=\sum_{i}^{p} \lambda_{i} v_{i}+\sum_{i}^{q} \gamma_{i} r_{i}, \sum_{i}^{p} \lambda_{i}=1,0 \leq \lambda_{i} \leq 1, \gamma_{i} \geq 0\right\}$.

Lemma 1. [9] Consider the system $x^{+}=A x+w$, with a Schur matrix $A$ and a non-negative vector $w$ such that $|w| \leq \bar{w}, \forall w \in \mathcal{W} \subset \mathbb{R}^{n}$. Let $A=V J V^{-1}$ be the Jordan decomposition of $A$. Then the set $\Omega_{U B}=\left\{x \in \mathbb{R}^{n}|| V^{-1} x \mid \leq\right.$ $\left.(I-|J|)^{-1}\left|V^{-1} \bar{w}\right|\right\}$ is robustly invariant (RI) with respect to the system's dynamics.

\section{PREREQUisites AND PROBLEM STATEMENT}

\section{A. Properties characterizing an agent}

Consider a leader-follower sub-system $\Sigma^{i}$ composed of the $i^{t h}$ agent as leader and its $N$ followers. $\mathcal{F}^{i}$ is the set of indices of the followers for the $i^{\text {th }}$ agent (identified by the existence of a connection to the node $i$ ). The nominal discrete-time dynamics equation of the $j^{\text {th }}$ follower is:

$$
x_{j}^{+}=A_{j} x_{j}+B_{j} u_{j}, j \in \mathcal{F}^{i}
$$

where $x_{j} \in \mathbb{R}^{n}$ and $u_{j} \in \mathbb{R}^{m}$ are respectively its nominal state vector and input vector. The pairs $\left(A_{j}, B_{j}\right)$ are assumed to be stabilizable, with $A_{j} \in \mathbb{R}^{n \times n}$ and $B_{j} \in \mathbb{R}^{n \times m}$. In the presence of disturbances, the model of the $j^{\text {th }}$ follower is considered to account for it additively:

$$
\tilde{x}_{j}^{+}=A_{j} \tilde{x}_{j}+B_{j} \tilde{u}_{j}+w_{j}
$$

where $w_{j} \in \mathcal{W}_{j}$ is the disturbance vector, $\tilde{x}_{j} \in \mathbb{R}^{n}$ and $\tilde{u}_{j} \in \mathbb{R}^{m}$ are the $j^{\text {th }}$ follower's real state and input vector, respectively. We assume that $\mathcal{W}_{j} \subset \mathbb{R}^{n}$ is bounded and contains the origin in its strict interior.

If the robust control input vector in (2) has the following form:

$$
\tilde{u}_{j}=u_{j}+K_{j}\left(\tilde{x}_{j}-x_{j}\right)
$$

then, by denoting $e_{j}=\tilde{x}_{j}-x_{j}$ as the tracking error of the $j^{t h}$ follower, we can obtain $e_{j}^{+}=\left(A_{j}+B_{j} K_{j}\right) e_{j}+w_{j}$.
By applying Lemma 1, a RPI set $\mathcal{S}_{j}$ is constructed (see [10]), ensuring that $e_{j}(t) \in \mathcal{S}_{j}$ at each considered time instant if $e_{j}(0) \in S_{j}$.

The set $\mathcal{S}_{j}$ is considered as a basic geometrical form for the safety region around the $j^{\text {th }}$ follower. Although the real state $\tilde{x}_{j}$ is unknown due to disturbances $w_{j}$, its trajectory is always bounded by the tube:

$$
\mathcal{S}\left(x_{j}\right)=\left\{x_{j}\right\} \oplus \mathcal{S}_{j}
$$

Therefore, the nominal dynamics (1) together with its robust tube-based safety region (4) is further used to designate a follower and furthermore an agent in general.

The collision avoidance constraint between two agents can be described via the exclusion of their safety regions i.e. $\mathcal{S}\left(x_{i}\right) \cap \mathcal{S}\left(x_{j}\right)=\emptyset$ or $x_{j}-x_{i} \notin \mathcal{S}_{j i}=\left\{-\mathcal{S}_{j}\right\} \oplus \mathcal{S}_{i}, \forall i \neq j$.

\section{B. Control design subject to collision avoidance constraints}

For the nominal sub-system $\boldsymbol{\Sigma}^{i}$, the local control of the $j^{t h}$ follower is $u_{j}=\bar{u}_{j}^{i}+K_{j}\left(x_{j}-\bar{x}_{j}^{i}\right)$, with $\bar{x}_{j}^{i}=\bar{x}_{j}+x_{i}$ and $\bar{u}_{j}^{i}=\bar{u}_{j}+u_{i}$. The pair $\left(\bar{x}_{j}, \bar{u}_{j}\right)$ denotes the admissible fixed point of the $j^{t h}$ follower determined in the neighborhood of the origin. Without loss of generality, the origin is considered to be the fixed point for the leader of $\boldsymbol{\Sigma}^{i}$. The pair $\left(\bar{x}_{j}, \bar{u}_{j}\right)$ are obtained by solving the following optimization

$$
\bar{x}_{j}^{*}=\underset{\bar{u}_{j}}{\operatorname{argmin}}\left\|\bar{x}_{j}\right\|
$$

subject to:

$$
\begin{aligned}
& \bar{x}_{j} \notin\left\{-\mathcal{S}_{j}\right\} \oplus \mathcal{S}_{i} \\
& \bar{x}_{j}=A_{j} \bar{x}_{j}+B_{j} \bar{u}_{j}
\end{aligned}
$$

The constraint $(5 c)$ characterizes the admissible points on the equilibrium hyperplane $\left(I_{n}-A_{j}\right) \bar{x}_{j}=B_{j} \bar{u}_{j}$, with the affine terms parametrized by $\bar{u}_{j}$. Moreover $\bar{x}_{j}=0$ if and only if $\bar{u}_{j}=0$ proving that the origin lies in this hyperplane. The expression $(5 \mathrm{~b})$ presents the collision avoidance constraint between the $j^{\text {th }}$ follower and its leader corresponding to the origin.

Fig. 1 shows the equilibrium hyperplanes and the fixed points of three followers around their leader $\bar{x}_{0}$. The convex polyhedrons represent their safety regions.

Remark 1. The expression (5b) can not guarantee the collision avoidance between this follower and its neighbors. To overcome this drawback, the fixed points of all followers in $\Sigma^{i}$ can be determined by the leader (see [3]). In the present paper, we consider only the case when the solutions of (5) do not violate the collision avoidance between the followers.

For the simplicity of notation, instead of determining the functioning zone relative to the current state of the leader, the problem can be translated without loss of generality to a positioning relative to the origin. Hence we can simplify the notations as $\bar{x}_{j}^{i}=\bar{x}_{j}$ and $\bar{u}_{j}^{i}=\bar{u}_{j}$ and rewrite the anti-collision constraint as

$$
x_{j} \notin \mathcal{S}_{j i}=\left\{-\mathcal{S}_{j}\right\} \oplus \mathcal{S}_{i}
$$




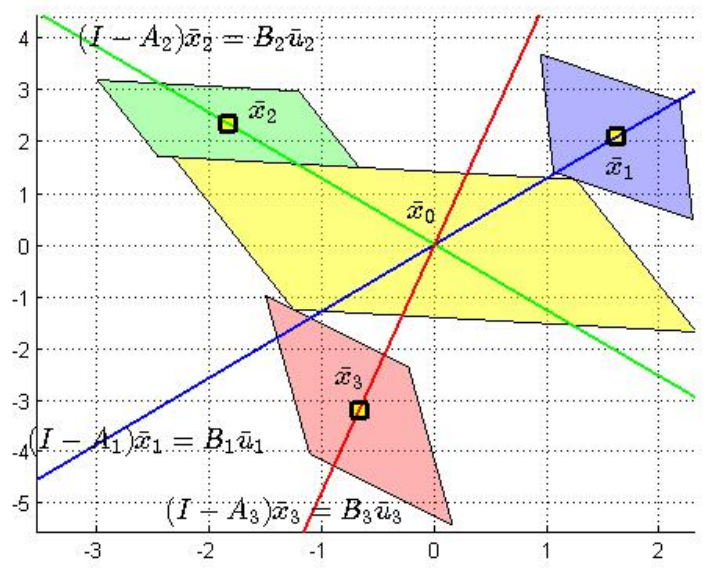

Fig. 1. Fixed points of 3 followers around the leader.

According to the property of the Minskowki sum, the set $\mathcal{S}_{j i}$ is convex and contains the origin.

The constraint (6) implies that the evolution of $x_{j}$ has to avoid the forbidden set $\mathcal{S}_{j i}$. In other words, $x_{j}$ will converge to a fixed point $\bar{x}_{j}$ on a supporting hyperplane $\{G x=\Gamma\}$ of the polyhedral set $\mathcal{S}_{j i}$ while avoiding the collision with $\mathcal{S}_{j i}$.

This requires to design a feedback control:

$$
u_{j}=K_{j}\left(\mathcal{H}_{j}\right) x_{j}
$$

which makes the half-space $\mathcal{H}_{j}=\left\{x \in \mathbb{R}^{n} \mid G x \geq \Gamma\right\}$ positive invariant and also stabilizes (1). $G x \leq \Gamma$ are the set of half-spaces forming the boundary of $\mathcal{S}_{j i}$ activated by $\bar{x}_{j}$. By construction, $\mathcal{H}_{j}$ belongs to the complement set of $\operatorname{int}\left(\mathcal{S}_{j i}\right)$.

The affine term $\Gamma$ can be neglected if the initial cone $\mathcal{H}_{j}$ is shifted to the origin (via a change of coordinates), leading to a proper cone.

According to [4], the linear ${ }^{1}$ control law (7) exists if and only if there exist a non-negative matrix $F_{j}$ and $P_{j}=P_{j}^{\top} \succ 0$ such that:

$$
\begin{aligned}
& G\left(A_{j}+B_{j} K_{j}\right)=F_{j} G \\
& {\left[\begin{array}{cc}
\delta P_{j} & \left(A_{j}+B_{j} K_{j}\right) P_{j} \\
P_{j}^{\top}\left(A_{j}+B_{j} K_{j}\right)^{\top} & P_{j}
\end{array}\right] \succeq 0}
\end{aligned}
$$

with the decision variables $F_{j}, K_{j}\left(\mathcal{H}_{j}\right)$ and $P_{j}$. The constraint (8a) expresses the invariance condition according to Definition 3 , while (8b) formulates the Lyapunov stability constraint, with $0<\delta<1$ the rate of convergence.

A method to solve (8) is detailed in [4]. In the present paper we start from the assumption that (8) is feasible.

Consider a polyhedral set $\mathcal{C}_{j}$ containing the origin and the local feedback control $u_{j}=K_{j}\left(\mathcal{H}_{j}\right) x_{j}$ defined over $\mathcal{C}_{j}$ and obtained by solving (8). Clearly, the local control $u_{j}$ is determined independently of $\mathcal{C}_{j}$. Hence, the entire set $\mathcal{C}_{j}$ is not control invariant but there still exists a control invariant

\footnotetext{
${ }^{1}$ In the general case, an affine control law can be used in order to handle fixed-points different from the origin.
}

subset $\Omega_{j} \in \mathcal{C}_{j}$. The set $\Omega_{j}$ can be characterized in terms of the Maximal Output Admissible Set (see [11]) associated with the local control $u_{j}=K_{j}\left(\mathcal{H}_{j}\right) x_{j}$. The algorithm to construct iteratively $\Omega_{j}$ is detailed in [11].

\section{Problem formulation}

In the centralized approach, all collision avoidance constraints have to be taken into account while designing the control strategy for the entire system. The number of these constraints and their non-convexity burn a considerable computation effort, making the centralized approach not suitable. As briefly mentioned in Section I, these drawbacks can be addressed by using a leader-follower structure. In this framework, the communication is restricted only between a follower and its leader. However, to ensure the collision avoidance, each follower has to operate within its restricted functioning zone. These zones do not intersect (in order to avoid collisions) and their union defines a partition of the working space.

This is particularly interesting for the plug-and-play control point of view because the partition is the only thing to be reconfigured when an agent leaves the formation (for instance due to a fault) or in case of integration of a new agent to the current formation (e.g. a recovered agent from a faulty situation).

However, this partition has to be suitable for the local dynamics of all the followers and also with the desired fixed points of the followers relative to the current leader's state. In other words, the local feedback control of a follower is determined with respect to the anti-collision constraints between its dynamics and the one of the leader. Thus not its entire functioning zone but only a region in the interior of the functioning zone is control invariant by the predefined feedback control. To construct such control invariant partition, we propose to design an interpolation-based control which drive all followers states towards their corresponding invariant zone and then switch to the local feedback control.

\section{INTERPOLATION BASED CONTROL}

The principles of the interpolation-based control were detailed in [12]. We extend this approach for a proper cone.

Consider a proper cone $\mathcal{H}$ and the maximal output admissible set $\Omega \subseteq \mathcal{H}$ associated with the linear time invariant dynamics (1) in the presence of a local control $u_{\Omega}=K x$. The control law $u$ is defined as a convex combination of $u_{\Omega}$ and a vertex control law associated with a $N$-step Robustly Controlled Set $\mathcal{P}_{N} \subseteq \mathcal{H}$. As a particularity, in the present case we would like to enforce $\mathcal{P}_{N}=\mathcal{H}$ but this goal can face a structural problem whenever the interpolation step has to deal with the unbounded functioning region $\mathcal{H}$ in conjunction with input constraints. In this case the feasible region for the interpolation scheme will be restricted to the maximal controllable set inside $\mathcal{H}$.

From the constrained control law computation, it will be considered that either $\mathcal{P}_{N}$ or a convex (polyhedral) controlled invariant subset $\Phi \supseteq \Omega$ is available together with a feasible 
control action on the boundaries of $\Phi$ denoted $u_{\Phi}$ (the socalled vertex control [13]). Therefore, the interpolated control action becomes

$$
u=\beta u_{\Phi}+(1-\beta) u_{\Omega}, 0 \leq \beta \leq 1
$$

where $\beta$ and $u_{\Phi}$ have to be calculated in real time. The component $u_{\Phi}$ will be activated when $x \in(\Phi \backslash \Omega)$ and the scalar $\beta$ has to be minimized under convex (polyhedral) constraints in order to get $u$ as close as possible to $u_{\Omega}$.

For simplicity, consider the set $\Phi$ scaled out from $\Omega$ with the restriction to the functioning set $\mathcal{H}$. The scaling of the set $\Omega$ will be done with respect to the fixed point $\bar{x}$ in order to preserve the control invariance of $\Phi$.

Proposition 1. An outer candidate set $\Phi$ for the interpolation is defined as $\Phi=\left(\{\bar{x}\} \oplus \mu \Omega_{0}\right) \cap \mathcal{H}$ with $\mu$ found by solving the following Linear Programming (LP) problem:

$$
\min _{\mu} \mu \text { s.t. }\left\{\begin{array}{l}
x \in\left(\{\bar{x}\} \oplus \mu \Omega_{0}\right) \\
\mu \geq 0
\end{array}\right.
$$

with $\Omega_{0}=\{-\bar{x}\} \oplus \Omega$ and $x$ the current state.

The construction procedure provides the two interpolation terms $\Phi$ and $\Omega$ for the constrained control design. We present next how to effectively obtain the vertex control action $u_{\Phi}$ and then the interpolation coefficient $\beta$.

The determination of the vertex control $u_{\Phi}$ exploits the fact that its objective is to push the state $x$ away from the boundary of $\Phi$ towards its interior, or alternatively $x^{+} \in \alpha \Phi$ with the minimal contraction factor $\alpha$. This can be done by solving the following LP problem:

$$
u_{\Phi}=\min _{\alpha, u_{\Phi}} \alpha \text { s.t. }\left\{\begin{array}{l}
A x+B u_{\Phi} \in \alpha \Phi \\
0 \leq \alpha \leq 1
\end{array}\right.
$$

Proposition 2. The optimization problem (11) is recursively feasible if $\mathcal{H}$ is control invariant.

Proof. The set $\Phi$ is defined as the intersection of two sets in Proposition 1 . The set $\bar{x} \oplus \mu \Omega_{0}$ is linearly control invariant by construction. If $\mathcal{H}$ is control invariant then the feasible set of (11) is the intersection of controlled invariant set and the problem is feasible.

For the determination of the scalar $\beta$, we exploit the fact that the control $u$ is obtained as an interpolation of $u_{\Omega}$ and $u_{\Phi}$ and has to be as close as possible to $u_{\Omega}$ which has a local linear control structure around $\bar{x}$. This desideratum can be translated in terms of an optimization problem (12). Consider $u=\beta u_{\Phi}+$ $(1-\beta) u_{\Omega}$, and $x$ decomposed as $x=\beta x_{\Phi}+(1-\beta) x_{\Omega}$, where $x_{\Phi} \in \Phi$ and $x_{\Omega} \in \Omega$. Let $\Phi=\left\{x \in \mathbb{R}^{n} \mid F_{\Phi} x \leq k_{\Phi}\right\}$ and $\Omega=\left\{x \in \mathbb{R}^{n} \mid F_{\Omega} x \leq k_{\Omega}\right\}$. Then the following optimization problem is obtained:

$$
\min _{\beta, x_{\Omega}, x_{\Phi}} \beta \text { s.t. }\left\{\begin{array}{l}
F_{\Phi} x_{\Phi} \leq k_{\Phi} \\
F_{\Omega} x_{\Omega} \leq k_{\Omega} \\
\beta x_{\Phi}+(1-\beta) x_{\Omega}=x \\
0 \leq \beta \leq 1
\end{array}\right.
$$

Although the problem (12) is nonlinear, we can translate it into a LP problem following the artifact proposed in [12], by using a change of variable $r=\beta x_{\Phi}$. Thus (12) becomes:

$$
\min _{\beta, r} \beta \text { s.t. }\left\{\begin{array}{l}
F_{\Phi} r \leq \beta k_{\Phi} \\
F_{\Omega}(x-r) \leq(1-\beta) k_{\Omega} \\
0 \leq \beta \leq 1
\end{array}\right.
$$

Solving respectively (10), (11) and (13) in real time provides the interpolation factor and implicitly the control $u=\beta u_{\Phi}+$ $(1-\beta) u_{\Omega}$.

We show the effectiveness of our idea in Fig. 2. The fixed point chosen is the origin. Given an initial state $x(0) \notin \Omega$, we solve firstly (10) to construct $\Phi$. Subsequently, we solve (11) to get $u_{\Phi}$ and further (13) to get $u$. We see that $x$ is driven from the boundary of $\Phi$ (yellow) into $\Omega$ (blue).

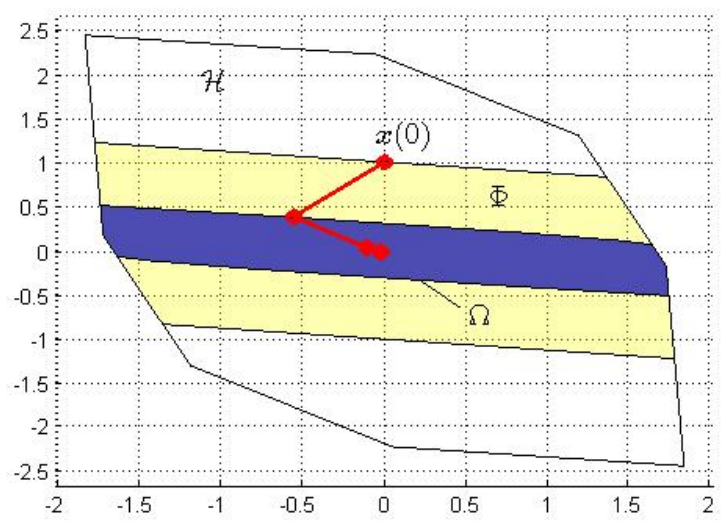

Fig. 2. Behavior of the of the $j^{t h}$ follower towards $\Phi$ and $\Omega$

The drawback of this procedure is that the feasibility of (11) is ensured if and only if the entire set $\Phi$ is control invariant. However from the definition of $\Phi$ in Proposition 1, the control invariance of $\Phi=\Omega \cap \mathcal{H}$ is not guaranteed. The main reason is that although $\Omega=\{\bar{x}\} \oplus\left(\mu \Omega_{0}\right)$ is control invariant, the control invariance of $\mathcal{H}$ is not granted. As underlined in Proposition 2 , the expression (11) is feasible if and only if $\mathcal{H}$ is control invariant. This leads to the idea of adjusting the given set $\mathcal{H}$ to guarantee the control invariance of the interpolation-based control strategy.

In our case, the set $\mathcal{H}$ represents a functioning zone $\mathcal{C}_{j}$, with $j \in \mathcal{F}^{i}$. Hence, we propose to guarantee the control invariance just for a subset in $\mathcal{C}_{j}$. This implies finding a partition appropriate to the local interpolation-based control in the functioning zone. A new method to deal with such requirement is presented in the next section.

\section{PARTitioning The FUnCTIONING ZONES}

In the sequel, we use the following definition of a partition for a Multi-Agent system:

Definition 5. Given a Multi-Agent sub-system $\boldsymbol{\Sigma}$ composed of $N$ agents, a partition $\mathfrak{P}[\boldsymbol{\Sigma}]$ for the state-space with respect to $\boldsymbol{\Sigma}$ is a set of $N$ polyhedral convex cells $\mathcal{C}_{j}$ which satisfy 
- $\mathcal{C}_{j} \cap \mathcal{C}_{k}=\emptyset, \forall j, k \in \mathcal{N}, j \neq k$;

- $\mathfrak{P}[\boldsymbol{\Sigma}]=\bigcup_{j \in \mathcal{N}} \mathcal{C}_{j}$;

- $\bar{x}_{j} \in \operatorname{int}\left(\mathcal{C}_{j}\right), \forall j \in \mathcal{N}$.

We will present in this section a methodology to construct a partition $\mathfrak{P}[\boldsymbol{\Sigma}]$. We recall that $\boldsymbol{\Sigma}$ contains an unique leader agent hence for brevity, the leader's index is neglected and we consider only the $N$ followers in $\boldsymbol{\Sigma}$. The set $\mathcal{N}$ contains their indices. A convex cell $\mathcal{C}_{i}$ in the Definition 5 corresponds to the functioning zone of the $i^{t h}$ follower.

Moreover, for simplicity, the current state of the leader is translated to the origin hence each functioning zone can take the form of a polyhedral proper cone. All of the following results are presented in $\mathbb{R}^{2}$ to fix the main steps in a formal manner. The extension for $\mathbb{R}^{p}$ with $p>2$ is currently under development. In the sequel, the following assumption is mentioned to simplify the presentation.

Assumption 1. $0_{n, 1} \in \operatorname{int}\left(\operatorname{Conv}\left\{\bar{x}_{i}, \forall i \in \mathcal{N}\right\}\right)$ and $\bar{x}_{i} \notin$ $\operatorname{int}\left(\operatorname{Conv}\left\{\bar{x}_{i}, \forall i \in \mathcal{N}\right\}\right)$.

This means that for the sub-system $\boldsymbol{\Sigma}$, the origin is in the strict interior of the convex hull of all fixed points $\bar{x}_{i}$ with $\forall i \in \mathcal{N}$ and there are no fixed points in the interior of the convex hull.

The cell $\mathcal{C}_{i}$ containing the $i^{\text {th }}$ agent state is defined as the linear combination of the hyperplanes which separate the $i^{t h}$ agent from its adjacent neighbors. Their construction is given in Proposition 3.

Proposition 3. The hyperplane used to separate a follower $i$ and its closest neighbor $j$ is the one passing through the origin and a point of separation $p_{a}$. This point is chosen as a linear combination of the equilibrium points $\bar{x}_{i}$ and $\bar{x}_{j}$ to ensure the separation, i.e.

$$
p_{a}=\theta_{a} \bar{x}_{i}+\left(1-\theta_{a}\right) \bar{x}_{j}
$$

with the scalar $0 \leq \theta_{a} \leq 1$. The index a belonging to the set of natural numbers $\Psi=\{1, \ldots N\}$ underlying that there is a correspondence between neighboring agents in $\mathcal{N}$, i.e. $a=a(i, j)$.

Consider now the $i^{\text {th }}$ agent with its two adjacent neighbors $j$ and $k$. The points chosen to separate them are respectively $p_{a}$ and $p_{b}$, with $a=a(i, j)$ and $b=b(j, k)$ belonging to $\Psi$. According to the Proposition 3, these points have to fulfill

$$
\left\{\begin{array}{l}
p_{a}=\theta_{a} \bar{x}_{i}+\left(1-\theta_{a}\right) \bar{x}_{j} \\
p_{b}=\theta_{b} \bar{x}_{j}+\left(1-\theta_{b}\right) \bar{x}_{k}
\end{array}\right.
$$

Therefore the cell $\mathcal{C}_{i}$ can be defined as a linear combination of unidirectional rays $\mathcal{C}_{i}=$ $\left\{x \in \mathbb{R}^{n} \mid x=\gamma_{a} p_{a}+\gamma_{b} p_{b}, \gamma_{a} \geq 0, \gamma_{b} \geq 0\right\}$.

For example, given $\boldsymbol{\Sigma}$ composed of 3 followers as illustrated in Fig.3. The indices set is $\mathcal{N}=\{i, j, k\}$. Based on their fixed points $\bar{x}_{i}$, the points of separation $p_{a}, p_{b}, p_{c}$ are determined with $a=a(i, j), b=b(j, k)$ and $c=c(k, i)$. Subsequently we get the partition $\mathfrak{P}[\boldsymbol{\Sigma}]$.

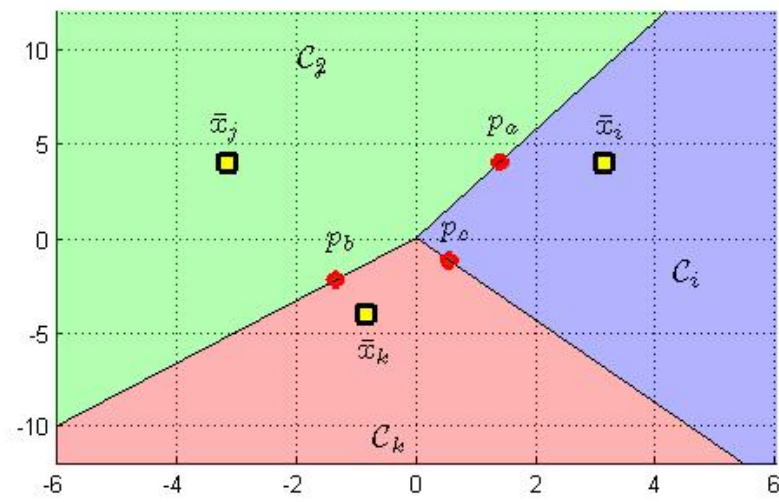

Fig. 3. Functioning zone assignment for 3 agents

We redefine the condition of invariance for $\mathcal{C}_{i}$ in terms of Definition 6

Definition 6. Consider a proper cone $\mathcal{C}=\left\{x \in \mathbb{R}^{n} \mid x=\right.$ $\left.\sum \gamma_{i} r_{i}, \gamma_{i} \geq 0\right\}$, with $r_{i}$ denoting an unidirectional ray. $\mathcal{C}$ is positively control invariant with respect to the system dynamics $x^{+}=A x+B u$ if and only if there exists a control $u$ and the non-negative scalars $\theta_{i} \geq 0$ such that $A x+B u=\sum_{i} \theta_{i} r_{i}$.

According to the Definition 6, the control invariance of two half-spaces sharing a common boundary passing through the origin and the point of separation $p_{a}$ is guaranteed if and only if there exist the non-negative scalars $\rho_{a i}, \rho_{a j}, \sigma_{a i}, \sigma_{a j} \geq 0$ and the control action $v_{a i}$ and $v_{a j}$ such that

$$
\left\{\begin{array}{l}
A_{i} p_{a}+B_{i} v_{a i}=\rho_{a i} p_{a}+\sigma_{a i} \bar{x}_{i} \\
A_{j} p_{a}+B_{j} v_{a j}=\rho_{a j} p_{a}+\sigma_{a j} \bar{x}_{j}
\end{array}\right.
$$

Let denote $\hat{\mathbf{A}}_{a}=\operatorname{col}\left\{A_{i}, A_{j}\right\}, \hat{\mathbf{B}}_{a}=\operatorname{diag}\left\{B_{i}, B_{j}\right\}$ and $v_{a}=\operatorname{col}\left\{v_{a i}, v_{a j}\right\}$. We can rewrite (15) and (16) for all the followers in $\boldsymbol{\Sigma}$, i.e.

$$
\begin{aligned}
& \hat{\mathbf{A}} \mathbf{p}+\hat{\mathbf{B}} \mathbf{v}=\Delta \mathrm{p}+\Gamma \overline{\mathbf{x}} \\
& \hat{\mathbf{x}}=\boldsymbol{\Theta} \overline{\mathbf{x}}
\end{aligned}
$$

with $\hat{\mathbf{A}}=\operatorname{diag}\left\{\hat{\mathbf{A}}_{a}\right\}, \hat{\mathbf{B}}=\operatorname{diag}\left\{\hat{\mathbf{B}}_{a}\right\}, \mathbf{v}=\operatorname{col}\left\{v_{a}\right\}$ and $\mathbf{p}=\operatorname{col}\left\{p_{a}\right\}$, with $\forall a \in \Psi, \overline{\mathbf{x}}=\operatorname{col}\left\{\bar{x}_{i}\right\}$, with $\forall i \in \mathcal{N}$.

The equations (17a) and (17b) extend respectively (16) and (15) for all the followers in the sub-system. The matrices $\boldsymbol{\Delta}$ and $\boldsymbol{\Gamma}$ are parameterized respectively by the scalars $\rho_{a i}, \rho_{a j}, \sigma_{a i}, \sigma_{a j}$ and $\theta_{a}$, i.e. $\boldsymbol{\Delta}=\boldsymbol{\Delta}\left(\left[\rho_{a i} ; \rho_{a j}\right]\right), \boldsymbol{\Gamma}=$ $\boldsymbol{\Gamma}\left(\left[\sigma_{a i} ; \sigma_{a j}\right]\right)$ and $\boldsymbol{\Theta}=\boldsymbol{\Theta}\left(\theta_{a}\right)$, with $\forall a \in \Psi$ and $\forall i, j \in \mathcal{N}$.

The problem (17) is non-convex because of the nonlinear product $\Delta$ p. However, the nonlinearity is mild in the sense that it involves bilinear terms and if this problem is feasible, the partition is control invariant and thus the feasibility of the interpolation-based control is guaranteed.

\section{ILLUSTRATIVE EXAMPLE}

Here we consider a heterogeneous sub-system $\Sigma$ composed of a leader and its three followers. The indices set of the 
followers is $\mathcal{N}=\{1,2,3\}$. The current position of the leader is replaced by the origin, thus its dynamics is neglected and it can be represented by its convex safety region centered in the origin. The followers dynamics is $x_{i}(t+1)=A_{i} x_{i}(t)+$ $B_{i} u_{i}(t)+w_{i}(t)$, with $A_{i}=\left[\begin{array}{cc}-0.2 & 0.5 \\ 0.2 & 0.71\end{array}\right], B_{1}=\left[\begin{array}{l}0.71 \\ 0.22\end{array}\right]$, $B_{2}=\left[\begin{array}{c}-0.71 \\ 0.22\end{array}\right], B_{3}=\left[\begin{array}{c}0.01 \\ -0.01\end{array}\right]$. The safety region is constructed by using a pole placement technique (choosing the poles $[0.2 ; 0.5])$. The disturbance is bounded, i.e. $\left|w_{i}\right| \leq$ $\left[\begin{array}{ll}0.2 & 0.2\end{array}\right]^{\top}$. The vector containing the fixed points for the three followers is $\overline{\mathbf{x}}=\operatorname{col}\left\{\bar{x}_{1}, \bar{x}_{2}, \bar{x}_{3}\right\}$, with $\bar{x}_{1}=[3.1123 ; 4]$, $\bar{x}_{2}=[-3.1443 ; 4]$ and $\bar{x}_{3}=[-0.8400 ;-4]$.

The closed-loop feedback control is calculated by each follower taking into account only the anti-collision constraints between the follower and the leader. Solving (8) for each follower gives the feedback gain $K_{1}=[-0.9091-0.8681]$, $K_{2}=\left[\begin{array}{lll}-0.9091 & 0.3472\end{array}\right]$ and $K_{3}=\left[\begin{array}{ll}20 & 1.0659\end{array}\right]$.

According to $\overline{\mathbf{x}}$, the entire working space is partitioned into three polyhedral proper cones $\mathcal{C}_{i}$ (as illustrated in Fig. 4), by solving (17) to obtain the control vector $\mathbf{v}$ and the points of separation $p_{a}=[1.3874 ; 4], p_{b}=[-1.3475 ;-2.2381], p_{c}=$ $[0.5454 ;-1.1957]$. The indices are expressed as $a=a(1,2)$, $b=b(2,3)$ and $c=c(3,1)$. Their maximal output admissible sets $\Omega_{i}$ are constructed according to the local control $u_{\Omega_{i}}=$ $\bar{u}_{i}+K_{i}\left(x_{i}-\bar{x}_{i}\right)$ and the functioning zone $\mathcal{H}=\mathcal{C}_{i}$, with $i \in \mathcal{N}$ (see Fig. 4).

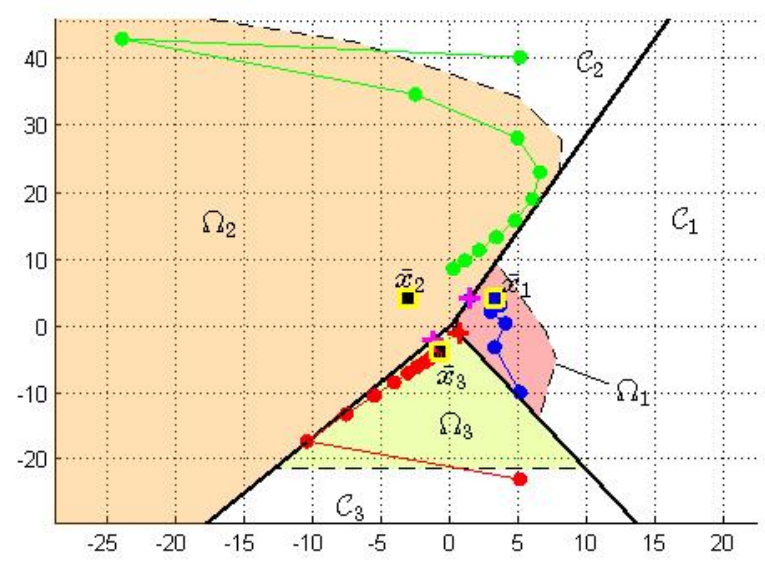

Fig. 4. Evolution of 3 agents towards their fixed points

We consider a set of initial points $x_{1}(0)=[5 ;-10]$, $x_{2}(0)=[5 ; 40]$ and $x_{3}(0)=[5 ;-23]$. The evolution of the three followers is shown in Fig. 4. For the $1^{\text {st }}$ agent (blue circle line), because $x_{1}(0) \in \Omega_{1}$, only its local control $u_{\Omega_{1}}$ is activated and it keeps $x_{1} \in \Omega_{1}$. For the $2^{\text {nd }}$ agent (green circle line) and the $3^{\text {rd }}$ agent (red circle line), $x_{2}(0) \notin \Omega_{2}$ and $x_{3}(0) \notin \Omega_{3}$, the interpolation based control is activated to push $x_{2}$ towards $\Omega_{2}$ and $x_{3}$ towards $\Omega_{3}$; subsequently the local control will be activated like the case of the $1^{\text {st }}$ agent.

\section{CONCLUSION AND PERSPECTIVES}

This paper presents a contribution to the decentralized control for a Muli-Agent System composed of multiple heterogeneous agents. The communication graph, relative to the dimension of the control design, is simplified by considering a leader-follower structure. This is often the solution to overcome the complexity drawbacks of the centralized approaches. Although the communication is reduced, the collision avoidance constraint can be still satisfied by partitioning the working space into multiple functioning zones. Subsequently, a control strategy based on interpolation techniques is proposed to drive the agent state towards the region where the local linear control is feasible. This requires a priori that the partition has to be control invariant. This paper presented the construction of such partition by means of solving an optimization problem. Its non-convexity needs to be further explored in future works in order to be able to characterize the global feasibility of the related system of constraints.

In the present work, the geometry of the agent's safety region and also the current state are not considered in the partition construction. Future work will also focus on these points because the partition needs to be updated in real time to be suitable to the current state of the agents (and thus improve the collision avoidance). Another interesting point is related to the existence condition of a partition if the origin is not included in the strict interior of the formation.

\section{REFERENCES}

[1] F. A. Fontes, D. B. Fontes, and A. C. Caldeira, "Model predictive control of vehicle formations," in Optimization and Cooperative Control Strategies. Springer, 2009, pp. 371-384.

[2] A. Grancharova and T. A. Johansen, "Distributed quasi-nonlinear model predictive control by dual decomposition," in Proceedings of the 18th IFAC World Congress, Milano, Italy, 2011, pp. 1429-1434.

[3] I. Prodan, "Commande sous contraintes de systemes dynamiques multiagents," Ph.D. dissertation, Supelec (in English), 2012.

[4] G. Bitsoris and S. Olaru, "Further Results on the Linear Constrained Regulation Problem," in Med. Control Conf., Crete, Greece, 2013.

[5] S. Rakovic, P. Grieder, and C. Jones, "Computation of Voronoi diagrams and Delaunay triangulation via parametric linear programming," ETH, Tech. Rep., 2004.

[6] E. Bakolas, "Optimal partitioning for multi-vehicle systems using quadratic performance criteria," Automatica, vol. 49, no. 11.

[7] F. Blanchini and S. Miani, Set-theoretic methods in control. Birkhauser, 2007.

[8] G. Bitsoris, "On the positive invariance of polyhedral sets for discretetime systems," Systems \& Control Letters, vol. 11, no. 3, 1988.

[9] E. Kofman, H. Haimovich, and M. M. Seron, "A systematic method to obtain ultimate bounds for perturbed systems," International Journal of Control, vol. 80, no. 2, pp. 167-178, 2007.

[10] I. Prodan, S. Olaru, C. Stoica, and S. Niculescu, "Predictive control for tight group formation of multi-agent systems," in Proc. of IFAC World Congress, Milan, Italy, 2011.

[11] E. Gilbert and K. T. Tan, "Linear systems with state and control constraints: the theory and application of maximal output admissible sets," IEEE TAC, vol. 36, no. 9, Sep 1991.

[12] H.-N. Nguyen, P.-O. Gutman, S. Olaru, and M. Hovd, "Implicit improved vertex control for uncertain, time-varying linear discrete-time systems with state and control constraints," Automatica, vol. 49, no. 9, 2013.

[13] P.-O. Gutman and M. Cwikel, "Admissible sets and feedback control for discrete-time linear dynamical systems with bounded controls and states," IEEE TAC, vol. 31, no. 4, pp. 373-376, 1986. 